\title{
Retrieving Atmospheric Profiles Data in the Presence of Clouds from Hyperspectral Remote Sensing Data
}

\author{
Xu Liu ${ }^{1}$, Allen M. Larar ${ }^{1}$, Daniel K. Zhou ${ }^{1}$, Susan Kizer ${ }^{1}$, Wan Wu ${ }^{1}$, Chris Barnet ${ }^{2}$, Murty Divakarla ${ }^{2}$, Guang \\ Guo $^{2}$, Bill Blackwell ${ }^{3}$, William L. Smith ${ }^{4}$, Ping Yang ${ }^{5}$, and Degui Gu ${ }^{6}$ \\ 1. NASA Langley Research Center, Hampton, VA 23666, USA \\ 2. NOAA Center for Satellite Applications, Camp Springs, MD 20746 USA \\ 3 MIT Lincoln Laboratory, Lexington, MA 02173 USA \\ 4. Hampton University, Hampton, VA 23668, USA \\ 5. Texas A\&M University, Department of Atmospherics Sciences, College Station, TX 77843, USA \\ 6.Northrop Grumman Aerospace Systems, Redondo Beach, CA \\ Xu.Liu-1@nasa.gov
}

\begin{abstract}
Different methods for retrieving atmospheric profiles in the presence of clouds from hyperspectral satellite remote sensing data will be described. We will present results from the JPSS cloud-clearing algorithm and NASA Langley cloud retrieval algorithm.

OCIS codes: (010.1280) Atmospheric composition; (010.5620) Radiative transfer
\end{abstract}

\section{Introduction}

Hyperspectral satellite sensors such as Infrared Atmospheric Sounding Interferometer (IASI) and Cross-track Infrared Sounder (CrIS) have high spectral and spatial resolution. They provide abundant information on atmospheric and surface properties. In order to retrieve atmospheric temperature, water, and trace gas vertical profiles from these high spectral resolution data, one has to account for cloud contributions to the top of atmospheric (TOA) radiance spectra. Usually there are two methods for dealing with clouds: cloud-clearing (CC) and cloudretrieval $(\mathrm{CR})[1,2,3,4]$. The $\mathrm{CC}$ is used by the AIRS level 2 data processing algorithm and by the Joint Polar Satellite System (JPSS) Cross-track Infrared and Microwave Sounder Suite (CrIMSS) algorithm. At NASA Langley Research Center (LaRC) [5,6,7,8,9], we have developed a retrieval algorithm, which explicitly retrieves cloud properties together with other parameters such as atmospheric temperature, moisture, and trace gases profiles, surface skin temperature and emissivity. We will present results of testing the CrIMSS Environmental Data Record (EDR) operational algorithm and the LaRC CR method using IASI satellite data.

\section{JPSS CrIMSS algorithm}

The operational CrIMSS EDR an algorithm was originally designed to run on large IBM computers with dedicated data management subsystem (DMS). We have ported the operational code to simple Linux systems by replacing DMS with appropriate interfaces. The ported code is capable of reading data from both the operational code and from the CrIMSS science code. The detail of the CrIMSS EDR algorithm is described in reference [1]. The cloudcleared radiance $\hat{R}_{i, c l r}$ for channel $i$ can be expressed as a linear combination of the measured radiances

$$
\hat{R}_{i, l r}=\bar{R}_{i, 1}+\eta_{1}\left[\bar{R}_{, 1}-\bar{R}_{i, K+1}\right]+\ldots+\eta_{k}\left[\bar{R}_{i, 1}-\bar{R}_{i,(K+2)-k}\right]+\ldots+\eta_{K}\left[\bar{R}_{i, 1}-\bar{R}_{i, 2}\right]
$$

Where $\eta_{1} \ldots \eta_{K}$ are unknown channel-independent constants, $\bar{R}_{i, 1} \ldots \bar{R}_{i, K+1}$ are the measured radiances and at least $\mathrm{K}+1$ field of views are needed to solve for K cloud formations. We will test the CRIMSS algorithm performance by generating proxy data from the IASI observations. The details will be given in the result section.

\section{NASA Langley cloud retrieval algorithm}

One of the uniqueness of the LaRC CR algorithm is that it uses a principal component based radiative transfer (PCRTM), which is capable of using all IASI spectral channels for the retrieval. It predicts projection coefficients of channel radiance onto a set of orthogonal eigenvectors (PCs), which capture the spectrum information in a descending order according to the associated eigenvalues. The channel radiance can be easily generated by a linear combination of PCs. The linear coefficients are called PC scores (or projection coefficients). Generally, only 100$200 \mathrm{PCs}$ are enough to regenerate the channel radiance to required accuracy. Since the dimension of the hyperspectral data is reduced from thousands to $\sim 100$, the computational cost for both forward radiative transfer calculations and inversions can be reduced dramatically. The inversion algorithm is based on a non-linear Levenberg-Marquardt method with climatology covariance and a priori information as constraints: 


$$
X_{n+1}-X_{a}=\left(K^{T} S_{y}^{-1} K+\lambda I+S_{a}^{-1}\right)^{-1} K^{T} S_{y}^{-1}\left[\left(y_{n}-Y_{m}\right)+K\left(X_{n}-X_{a}\right)\right]
$$

Where $x$ represents state vector, the subscripts $n$ and a priori, respectively. $K$ is the Jacobian matrix. $Y_{m}$ is the transformed observations (in this case an EOF transformed radiance spectrum). $Y_{n}$ is the forward model calculated PC scores using the state vector obtained from the nth iteration. $S_{y}$ and $S_{a}$ are error covariance matrices associated with observation (including forward model errors) and background state vector, respectively. All relevant parametes such as cloud properties, atmospheric profiles, and surface properties are retrieved simultaneously. The spatial resolution of the LaRC approach is higher than the CrIMSS EDR algorithm since it does not need to use multiple FOVs to perform cloud clearing.

\section{Results}

The IASI on board the METOP-A satellite provides all the information needed to generate CrIS proxy data. The IASI instrument is a Fourier Transform Spectrometer (FTS) with a spectral sample interval of $0.25 \mathrm{~cm}^{-1}$ and a continuous spectral coverage from 645 to $2760 \mathrm{~cm}^{-1}$. The CrIS instrument has three spectral bands with spectral coverage of $648.75-1096.25 \mathrm{~cm}^{-1}, 1207.5-1752.5 \mathrm{~cm}^{-1}$, and $2150.0-2555.0 \mathrm{~cm}^{-1}$, respectively [5]. The transformation from an IASI radiance spectrum to a CrIS proxy spectrum is a rigorous mathematical operation. The generated proxy data have been successfully ingested into the CrIMSS OPS code. This data set is very useful for algorithm testing and validation because it provides realistic scenes (atmosphere, surface and cloud), which are based on observations. It will help us identify areas of deficiencies in both forward and inverse models, testing robustness of the operational code, check error handling capability of the OPS code. It will be used for bias characterization and algorithm tuning, and EDR performance evaluations. Details of the results of this study will be presented at the conference.

We have applied the PCRTM radiative transfer model and inversion algorithm to hyperspectral sensors such as AIRS, IASI and NAST-I. The errors between the PCRTM calculated brightness temperature spectra and those calculated using line-by-line radiative transfer model are typically less than $0.05 \mathrm{~K}$, a value much smaller than the instrument noise. Figure 1 shows two IASI brightness temperature spectra, one observed by the IASI (red) and the other calculated using the PCRTM (blue). The cloud properties are retrieved using the method described in the previous section. The retrieval algorithm identifies the cloud phase as ice. The retrieved cloud top pressure is at 273 $\mathrm{hPa}$. The cloud effective size is $38 \mathrm{~mm}$ and the cloud visible optical depth is 0.462 . The bottom panel of the figure shows the difference between the observed and the calculated IASI spectra. They agree with each other within the IASI instrument noise levels (shown in red lines) in most of the spectral regions.

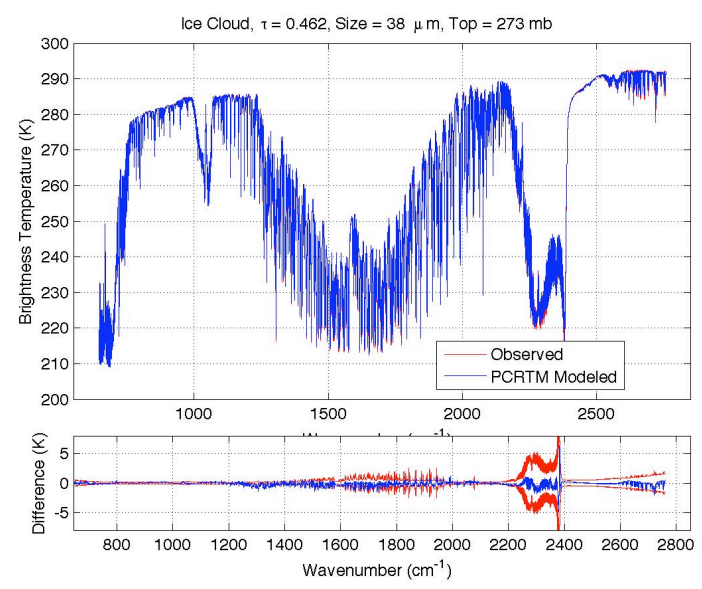

Figure 1. Top: Observed IASI spectrum and the PCRTM calculated spectrum. Bottom panel: Difference between observed and calculated IASI spectra (blue curve) and the IASI instrument noise converted to brightness temperature unit (red curves).

Figure 2 shows retrieved global monthly mean (July 2009) temperature at 300 mbar (top left), surface skin temperature (top right), surface emissivity at $1140 \mathrm{~cm}^{-1}$ (bottom left), and carbon monoxide mixing ratio near 500 mbar. The global distributions of atmospheric temperature and surface skin temperature agree well the ECMWF monthly means. The temperatures are high in the tropical region and low in polar region with regional and seasonal 
variations well captured by the retrieval system. Studies done in the past have shown that the retrieved temperature and moisture profiles agree well with collocated radiosondes. The surface emissivity retrieval provides emissivity spectrum at each IASI footprint when the cloud is not thick. The map shown in this figure clearly displays the low emissivity values of sand features in desert regions. The $\mathrm{CO}$ mixing ratios are elevated near either pollution sources (southeast Asia) or biomass burning sources (central Africa).
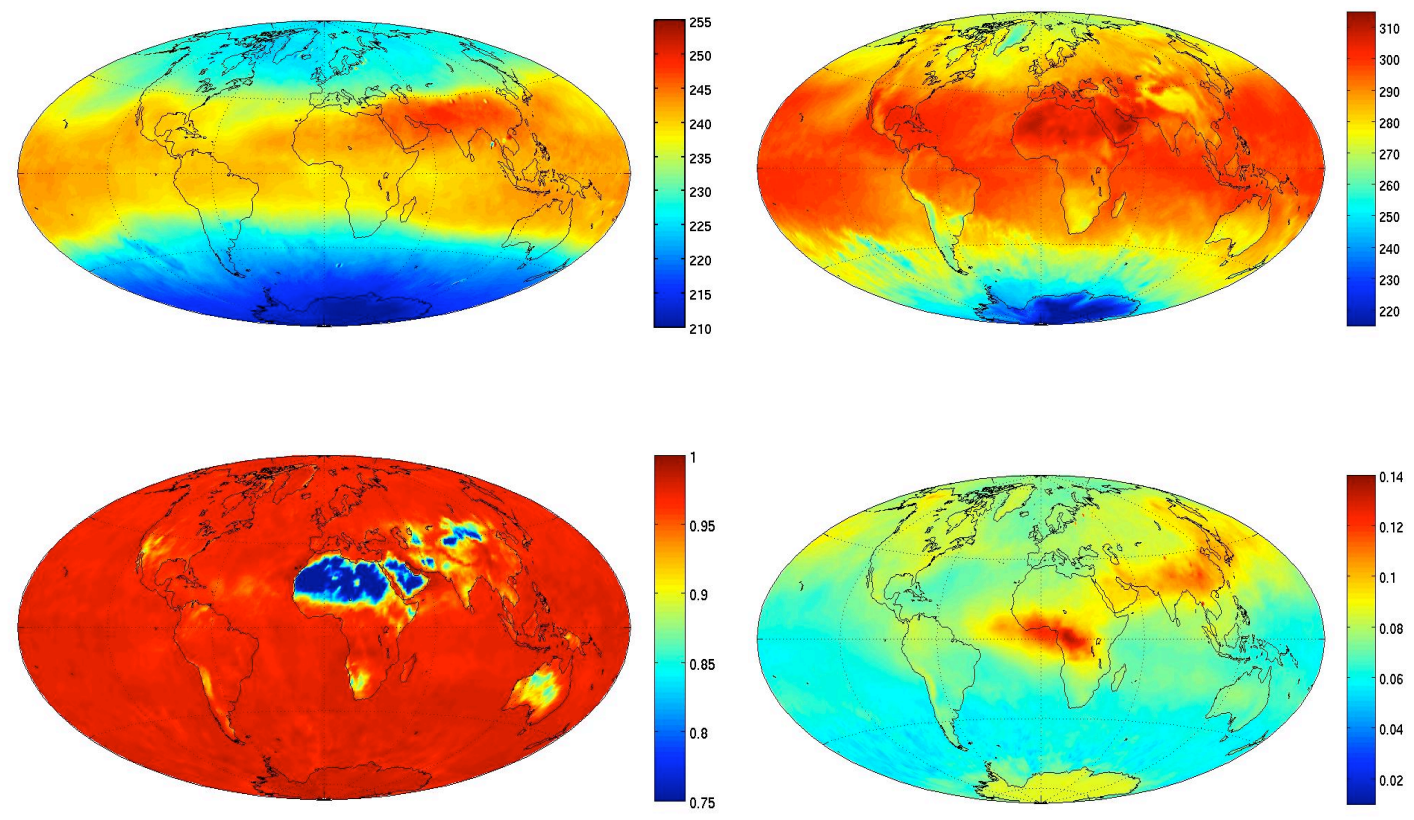

Figure 2. Retrieved global monthly mean (July 2009) temperature at 300 mbar (top left), surface skin temperature (top right), surface emissivity at $1140 \mathrm{~cm}-1$ (bottom left), and carbon monoxide mixing ratio near $500 \mathrm{mbar}$.

\section{Conclusions}

In preparation for the launch of NPP satellite, we have tested the CrIMSS operational code using proxy data generated from the IASI spectra. The CrIMSS EDR algorithm works well with the proxy data. We have also tested another algorithm developed at NASA Langley Research Center to retrieve atmospheric profiles along with cloud parameters. The method has been successfully applied to the IASI spectra directly.

\section{References}

[1] Northrop Grumman Space Technology, "Cross Track Infrared Sounder (CrIS) Volume II, Environmental Data Records (EDR) Algorithm Theoretical Basis Document ATBD”, Northrop Grumman Space Technology, Redondo Beach, CA, Feb. 8, 2007. http://jointmission.gsfc.nasa.gov/science/sciencedocuments/072010/D43772_Rev\%20C_CrIS_CrIMSS_ATBD_Final_Dist\%20ECR\%20A-

120A_Statement $\% 20 \mathrm{~A} \% 20$ Submission.pdf

[3] X. Liu, and S. Kizer, "Testing CrIMSS EDR Algorithm Using Synthetic and Proxy Data", Sounder Operational Algorithm Team (SOAT) Meeting, Logan, UT, September 10, 2009. ftp://www.star.nesdis.noaa.gov/pub/smcd/spb/nnalli/SOAT/2009-09/090910soat_04_04_liu.pdf

[4] M. T. Chahine, M. Gunson, et.al., "AIRS Level 2 Algorithm Theoretical Basis Document Version 2.2”, JPL D-17006, Version 2.2, April 26, 2001

[5] EUMETSAT, "IASI Level 1 Product Guide”, EUM/OPS-EPS/MAN/04/0032, Issue v3, May 13, 2009

[6] Liu X, Smith WL, Zhou DK, Larar A, "A principal component-based radiative transfer forward model (PCRTM) for hyperspectral instruments", Proc. SPIE Int. Soc. Opt. Eng. 5655, 96-105 (2005)

[7] Liu X, Smith WL, Zhou DK, Larar A, "Principal component-based radiative transfer forward model (PCRTM) for hyperspectral sensors: Theoretical concept", Appl. Opt. 45, 201-209 (2006)

[8] Liu, X., D. K. Zhou, Allen Larar, W. L. Smith, and S. A. Mango, 2007: Case Study a New Radiative Transfer Model and Retrieval Algorithm using EAQUATE Data, Q. J. R. Meterol. Soc. 133, 243-256.

[9] Liu, X., D. K. Zhou, A. M. Larar, W. L. Smith, P. Schlüssel, S. Newman, J. P. Taylor, and W. Wu, 2009a, "Retrieval of atmospheric profiles and cloud properties from IASI spectra using super-channels," Atmos. Chem. Phys., vol. 9, pp. 9121-9142, Dec. 2009. 